\title{
HIGH RESOLUTION LASER TECHNIQUES APPLIED TO REACTION DYNAMICS
}

\author{
RAYMOND J. VETTER \\ Laboratoire Aimé Cotton. C.N.R.S. II. 91405 Orsay Cedex, France
}

The applications of high resolution laser techniques to crossed-beam experiments which are examined in this paper concern: (a) the reactivity of atomic/molecular excited states and the influence of light polarization and (b) the measurement of absolute and differential cross sections by use of the laserinduced fluorescence technique performed in a coherent saturation regime.

Examples are provided by the crossed-beam study of the Cs(7P) $+\mathrm{H}_{2} \rightarrow \mathrm{CsH}+\mathrm{H}$ photochemical reaction, where two C.W. single-mode tunable lasers are used to excite Cs atoms and to probe $\mathrm{CsH}$ product molecules. It is shown how the fine and hyperfine structures of the Cs atom are involved in the reaction mechanism (harpooning). The measurement of the total cross section $\left(\approx 4.10^{-16} \mathrm{~cm}^{2}\right)$ gives an indication of the overall efficiency of the reactive process (Cs atoms in the 7P state) with respect to the quenching ones (Cs atoms in $6 \mathrm{~S}, 6 \mathrm{P}, 5 \mathrm{D}$ and $7 \mathrm{~S}$ states). Finally, the differential cross section shows a marked forward "peaking" of $\mathrm{CsH}$ products which narrows with collision energy but which is rather independent of the product rotation.

KEY WORDS: High resolution, laser reaction, dynamics.

\section{INTRODUCTION}

The major aim of reaction dynamics is to observe and to understand the intimate details of chemical reactivity, at the most elementary level of individual, isolated collisions between partners of well-defined energy and orientation. Reaction dynamics involves small systems and simple models which can be extrapolated to reactions of chemical, biological or industrial interest. Selectivity is essential in the driving of reactions: Excitation of reagents, breaking of chemical bonds, trapping of undesired products. In that respect, lasers are efficient tools to bring selective energy but their use is limited to small systems, the energy deposited in large ones being rapidly randomized. On an other hand, up to now, reliable calculations of potential energy and collision dynamics are possible for reactive systems involving a limited number of atoms and electrons.

The crossed-beam experiment is the selected device to study reaction dynamics in gas phase since it allows for the control of the collision geometry and for the probe of products in their nascent states. Furthermore, by use of supersonic expansions, it is possible to reduce the velocity spread of reagent molecules and to concentrate them in their lowest energy states. In this manner, many results were collected which demonstrated the principal features of reaction dynamics and led to the development of collision models, for systems in their ground state mainly. ${ }^{1}$ The advent of tunable 
lasers enlarged the field to the study of excited states and photon-induced processes. ${ }^{2}$ Beside the systematization, specific studies could be performed. In addition, two techniques of detection were developed and widely used: The laser-induced fluorescence and the resonance-enhanced multiphoton ionization which provided both a comfortable increase of the energy resolution.

Conditions are thus met together to approach the "ideal" experiment where all parameters are under control and to achieve state-to-state analyses of simple reactions. Unfortunately, the impact parameter is still out of control, a major drawback which is inherent to this type of experiment. It can be overcome in some particular experiments, where the photoexcitation of a Van der Waals complex is followed by its dissociation, the so-called "half-collision" having a fixed impact parameter and a well-defined geometry. ${ }^{3}$ Also, the present type of experiment does not yield direct information about the transition state. Difficult experiments were performed in that direction, ${ }^{4}$ but the major advance has arised recently with the development of real-time femtochemistry, where ultrashort laser pulses are used to follow the photodissociation of simple molecules. ${ }^{5}$ Application of this technique to the study of bimolecular collisions under crossed-beam conditions is not yet possible. On an other hand, by its very nature, femtochemistry is not sensitive to small energies. In that respect, it may be considered at the opposite of high resolution experiments performed with narrow-band C.W. lasers.

Some applications of high resolution laser techniques to crossed-beam experiments are presented here, with particular emphasis on the $\mathrm{Cs}(7 \mathrm{P})+\mathrm{H}_{2} \rightarrow \mathrm{CsH}+\mathrm{H}$ reaction: Selective excitation of reagents and measurement of total and differential cross sections.

\section{SELECTIVE EXCITATION OF REAGENTS}

The selective excitation of reagents which can be realized in crossed-beam experiments opens the field of reaction dynamics to photochemistry, thus to a possible generalization of the concepts. Beside, specific effects can be studied, those connected with the polarized nature of the laser field for instance. The following points are considered, according to the nature of the reagents (atoms/molecules) and to the characteristics of the laser field (monochromatically monochromaticity polarization).

\section{Monochromatic Excitation of Atomic/Molecular Reagents}

Many results have been obtained in this field. Lifetime and density of excited states are of course important parameters. Short-lived states must be excited at the collision volume (then, underlying excited states can also participate to the reaction) whereas metastable states can be excited upstream the collision volume (then, short-lived states have time to decay). Estimating the density of excited states is a difficult challenge to cross section measurements. The upper limit is half that of the ground state for a two-level system at complete saturation, it can be much lower (several percent) if the excited level is efficiently connected with many underlying 
ones. The selective excitation of reagents can result into two effects that it may be difficult to separate. First, the increase of potential energy can lead to the overcome of potential barriers or to the switching of endoergic reactions and, second, the choice of a specific quantum state can be a deciding factor since reagents and products of the same symmetry generally correlate on the reaction path.

Many examples concern reactions with excited atoms. ${ }^{6}$ For $\mathrm{Hg}\left({ }^{3} \mathrm{P}_{2,0}^{0}\right)+\mathrm{Br}_{2} \rightarrow \mathrm{HgBr}+\mathrm{Br}$, the higher fine structure level is more reactive ${ }^{7}$ whereas for $\mathrm{Br}\left({ }^{2} \mathrm{P}_{1 / 2,3 / 2}\right)+\mathrm{IBr} \rightarrow \mathrm{Br}_{2}+\mathrm{I}$, the lower level is more reactive by a factor $20 .{ }^{8}$ For $\mathrm{Na}+\mathrm{HCl} \rightarrow \mathrm{NaCl}+\mathrm{H}$, differential cross sections depend on the atom initial state: $3 \mathrm{~S}, 3 \mathrm{P}, 4 \mathrm{D} .{ }^{9}$ For $\mathrm{Ba}+\mathrm{O}_{2} \rightarrow \mathrm{BaO}+\mathrm{O}$, product energy distributions depend on the atom multiplicity, ${ }^{1} \mathrm{D}_{2}$ or ${ }^{3} \mathrm{D}_{2}$ in the $6 \mathrm{~s} 5 \mathrm{~d}$ electronic configuration. ${ }^{10}$ For $\mathrm{Ca}+\mathrm{N}_{2} \mathrm{O} \rightarrow \mathrm{CaO}+\mathrm{N}_{2}$, the yield of chemiluminescence decreases with collision energy for $\mathrm{Ca}\left({ }^{1} \mathrm{D}\right)$ but increases for $\mathrm{Ca}\left({ }^{3} \mathrm{P}\right) .{ }^{11}$

Vibrational excitation of molecules was considered at the very beginning of reaction dynamic studies. It gives rise to the so-called "vibrational enhancement", showing the ability of vibrational excitation to overcome a potential barrier or to compensate the endoergiticity (a generalization is found in Polamyi's rules ${ }^{12}$ ). Examples are provided by the bulk study of $\mathrm{Br}+\mathrm{HCl}\left(v^{\prime \prime}\right) \rightarrow \mathrm{HBr}+\mathrm{Cl}$, where an enhancement of the cross section of 11 orders of magnitude was measured between $v^{\prime \prime}=0$ (the reaction is endoergic) and $v^{\prime \prime}=2$ (it is exoergic), ${ }^{13}$ by the crossed-beam study of $\mathrm{K}+\mathrm{HCl}\left(v^{\prime \prime}\right) \rightarrow \mathrm{KCl}+\mathrm{H}$, where an enhancement of two orders of magnitude was observed between $v^{\prime \prime}=0$ and $v^{\prime \prime}=1 .{ }^{14}$ On the contrary, rotational excitation has led to a few contradictory results. The $\mathrm{K}+\mathrm{HF} \rightarrow \mathrm{KF}+\mathrm{H}$ system shows a little increase of the cross section with rotation, whereas $\mathrm{K}+\mathrm{HCl} \rightarrow \mathrm{KCl}+\mathrm{H}$ shows a steep decrease. ${ }^{15}$

It is worthwhile to mention here that a new technique using two lasers to excite a three-level system in a molecular beam has been developed recently, which authorizes a very efficient transfer of population (approaching 100\%!) from a rovibrational level of the ground state to another level of the same electronic state. ${ }^{16}$ It opens the way to systematic studies of vibrational and rotational effects.

\section{Polarization Effects in Atomic/Molecular Excitation}

In crossed-beam experiments performed with "aligned" atoms or "oriented" molecules, the rotation of the laser polarization with respect to the collision axis may induce striking new effects. This was first demonstrated on $\mathrm{Ca}\left({ }^{1} \mathrm{P}_{1}\right)+\mathrm{HCl} \rightarrow \mathrm{CaCl}\left(\mathrm{B}^{2} \Sigma^{+}, \mathrm{A}^{2} \Pi\right)+\mathrm{H}$, for which the yield of the $\mathrm{B}^{2} \Sigma^{+}$state is favorized when the atomic orbital is parallel to the collision axis and that of $A^{2} \Pi$ when it is perpendicular, ${ }^{17}$ for $\mathrm{Na}(4 \mathrm{D})+\mathrm{HCl} \rightarrow \mathrm{NaCl}+\mathrm{H}$, the cross section is larger when the $d$ orbital is parallel to the collision axis. ${ }^{9}$ Since these pioneering studies, the field has greatly developed. ${ }^{18}$

However, the real world of "stereochemistry" concerns reactions where the reagent molecules have well-defined orientations. This was realized first by $\mathrm{R}$. B. Bernstein, in the crossed-beam study of $\mathrm{Rb}+\mathrm{ICH}_{3} \rightarrow \mathrm{RbI}+\mathrm{CH}_{3}$, where electric fields are used to focus, to select and to orientate symmetric top molecules in 
well-defined quantum states. ${ }^{1} \mathrm{He}$ showed the existence of a cone of unfavourable attack angles within which there is a negligible probability to form RbI products. Partial alignment of excited molecules in the laboratory frame is also possible by using linearly-polarized laser light, since the molecular absorption is proportional to $\left|\mu . E_{0}\right|^{2}$, where $\mu$ is the molecular transition dipole and $E_{0}$ the amplitude of the laser electric field. This is illustrated by experiments on the $\mathrm{K}+\mathrm{HF}$ system, where the comparison of "parallel" and "perpendicular" cross sections gives information on the potential. ${ }^{15}$

\section{L.I.F. MEASUREMENTS OF TOTAL AND DIFFERENTIAL CROSS SECTIONS}

For a $\mathrm{A}+\mathrm{BC} \rightarrow \mathrm{AB}+\mathrm{C}$ reaction, the total cross section is given by the usual expression: ${ }^{1}$

$$
\sigma=N_{\mathrm{AB}}\left(n_{\mathrm{A}} n_{\mathrm{BC}} v \Delta V\right)^{-1}
$$

The $n$ stand for reagent densities, $v$ for the reagent relative velocity, $\Delta V$ for the collision volume. They can be determined by standard techniques. ${ }^{1} N_{\mathrm{AB}}$ is the number of products created per second in $\Delta V$. Its determination depends on the characteristics of the detection technique.

In the laser-induced fluorescence technique (L.I.F.), product molecules in a given nascent state absorb the laser beam at their resonance frequency and, once excited, give rise to fluorescence emission. This fluorescence is emitted isotropically, its intensity is proportional to the absorption coefficient (thus to the population of the level which is probed) and its spectral shape is linked to the Doppler profile associated with product recoil velocities. Indeed, the use of narrow-band tunable lasers allows for the high resolution recording of these profiles whose analysis gives two results: The product density and the product angular scattering probability. However, in general, the L.I.F. signal is not proportional to the laser density of energy because saturation effects readily occur. This is particularly true in the case of pulsed lasers but also in the case of usual C.W. tunable dye lasers which carry a much weaker energy.

For a C.W. laser field $E_{0}$ interacting with a two-level system, a "coherent saturation regime" is achieved when the interaction time (or transit time) $\Delta t$ of the product molecules with the laser beam is long compared with their lifetime $\tau$ in the excited level. ${ }^{19}$ Rate equations involving only level populations are not enough to describe the interaction and one has to introduce optical coherences between the two levels to solve the Schrödinger equation and to get the evolution of populations.

The strength of the interaction is characterized by the Rabi frequency:

$$
f_{1}=E_{0} h^{-1}\langle b|D . \varepsilon| a\rangle
$$

where $D$ is the dipole moment of the transition and $\varepsilon$ the polarization vector. In the case where a small fraction $(<10 \%)$ of the excited molecules return to the initial level ("open" system), the interaction of the product molecules at rest with the laser beam 
can be described by a resonant function $I(v)^{19}$ whose shape and width are function of $f_{1}$ and $\Delta t . I(v)$ is the main component to the apparatus function of the experimental set-up; at a very low saturation, it is a Lorentzian of width $\Gamma=1 / 2 \pi \tau$.

$N_{\mathrm{AB}}$ is determined from the total number $N$ of photons collected by the photodetector during the scanning of the laser frequency over the product absorption profile. $N$ is proportional to $N_{\mathrm{AB}}$ through characteristic parameters of the set-up: The efficiency of the detection optics, the speed of the frequency scanning, the shape and width of $I(v)$. However, since $I(v)$ is resonant with the laser frequency, only its width is important. It can be approximated from the previous molecular parameters, from the width of narrow Doppler-free resonances recorded under saturated-absorption conditions,${ }^{20}$ or from the width of the apparatus function which constitutes an upper limit. In addition, the variation of the interaction time within the collision volume reduces the efficiency of the detection. Consequently, one is led to calculate an "effective" interaction function to take these effects into account. ${ }^{21,22}$

The L.I.F. technique has been seldom used to measure reactive cross sections. In principle, it is possible to detect one photon per second, thus it should be possible to detect one product molecule created per second at the collision volume but, in practice, this is not the case. For most crossed-beam experiments, the optics efficiency is low since optical filters are required to eliminate stray light in the collision chamber; it is further reduced when reagents are excited at the collision volume and emit a parasitic fluorescence. By contrast, the resonance-enhanced multiphoton ionization technique is more sensitive since the collection of ions is very efficient, but it is strongly non-linear by its very nature. ${ }^{1}$

High resolution analyses of laser-induced fluorescence profiles can yield total state-to-state cross sections, they can also yield differential cross sections, as is shown now.

The problem is to determine in the center-of-mass (c.m.) the probability $P(w, \Theta)$ of reaction products to scatter with the recoil velocity $w$, at the angle $\Theta$ with respect to the collision axis. The method was suggested by J. L. Kinsey, in $1977 .{ }^{23}$ Its principle is to measure the frequency shift $\delta v$ of product molecules when they absorb a laser beam at the frequency $v$. The Doppler relation gives:

$$
\delta v=v_{0}-v_{0}=v_{0} w \cos \alpha / c
$$

where $v_{0}$ is the resonance frequency, $\alpha$ the angle between $w$ and the beam axis.

Scanning the laser frequency yields a Doppler profile, $D\left(v-v_{0}\right)$, whose shape depends on $P(w, \Theta)$ but not on the velocity of the c.m. which gives a constant (or null) frequency shift. Extracting $P(w, \Theta)$ from $D\left(v-v_{0}\right)$ is not trivial because the shape of $D\left(v-v_{0}\right)$ depends also on the orientation of the laser beam with respect to the collision axis. Two beam arrangements were proposed a decade $\mathrm{ago}^{24}$ and applied successfully to inelasting ${ }^{25}$ scattering and reactive ${ }^{26}$ experiments.

In a parallel arrangement, the laser beam propagates in the collision plane, along the collision axis: $\alpha \equiv \pi-\Theta$, and the correspondence is unique between $v$ and $w \cos \Theta$. Calculations show that:

$$
D\left(v-v_{0}\right)=2 \pi N c\left(v_{0} w\right)^{-1} P(w, \Theta)
$$


If $w$ is unique, the recording of $D\left(v-v_{0}\right)$ yields directly $P(\Theta)$, thus the differential cross section $d \sigma / d \Omega$; the angular resolution, $d v / d \Theta$, is best for $\Theta$ values around $90^{\circ}$ This situation holds in crossed-beam state-to-state studies of $\mathrm{A}+\mathrm{BC} \rightarrow \mathrm{AB}+\mathrm{C}$ reactions, where $\mathrm{AB}$ products are probed in a given $(v, J)$ quantum state and have a unique recoil velocity. If recoil velocities are spread over a narrow distribution, the scattering angles are smeared out resulting in a limitation of the angular resolution. In other cases, an $\mathrm{AB}+\mathrm{CD} \rightarrow \mathrm{AC}+\mathrm{BD}$ exchange reaction for example, the recoil velocity of $\mathrm{AC}$ (or $\mathrm{BD}$ ) products may not be unique, even if they are probed on a given quantum state. Then, Doppler profiles result in a superposition of several components of different shape and width.

In a perpendicular arrangement, the laser beam is adjusted perpendicularly to the collision plane, thus to the collision axis. The correspondence between $\Theta$ and $v$ is not unique because $\Theta$ is also a function of the azimuthal angle $\phi$ (the angles are connected by the relation: $\Theta=\operatorname{Arccos}[\sin \alpha \cos \phi])$. A spectral element of $D\left(v-v_{0}\right)$ at $v$ (thus at $\alpha$ ) results from the contribution of particles which scatter at all $\Theta$ values between $(\pi / 2)-\alpha$ and $(\pi / 2)+\alpha$. Calculations show that:

$$
D\left(v-v_{0}\right)=-N c\left(v_{0} w\right)^{-1} \int P[w, \Theta(\alpha, \phi)] d \phi
$$

The integral is calculable by numerical methods, or by a mathematical inversion of the Doppler profile. ${ }^{24}$ In any case, $D\left(v-v_{0}\right)$ is symmetrical with respect to $v_{0}$ since products scatter symmetrically with respect to the collision plane: With this beam arrangement, the profile analysis can only provide the $\operatorname{sum} P(w, \Theta)+P(w, \pi-\Theta)$. The angular resolution is best for $\Theta=0$ or $180^{\circ}$.

The first arrangement is difficult to apply to crossed-beam experiments because it provides much stray light in the collision chamber; furthermore, the adjustment of the laser beam along the collision axis must be performed for each collision energy. The perpendicular arrangement, which does not lead directly to $P(w, \Theta)$, is much easier to handle and is widely used. The two arrangements are thus more complementary than contradictory. They have been used to measure reactive differential cross sections for two reactions only: $\mathrm{Cs}+\mathrm{H}_{2}{ }^{27}$ and $\mathrm{I}_{2}+\mathrm{F}^{28}$

\section{THE Cs(7P) $+\mathrm{H}_{2} \rightarrow \mathrm{CsH}+\mathrm{H}$ REACTION}

This reaction involves only three atoms and three electrons, making it possible to calculate potential energy surfaces and collision dynamics. The reaction endoergiticity $(\approx 2.7 \mathrm{eV})$ can be compensated by the $6 \mathrm{~S}_{1 / 2} \rightarrow 7 \mathrm{P}_{1 / 2,3 / 2}$ excitation of Cs atoms. Then, the reactive system is just at threshold. As the $\mathrm{CsH}\left(X^{1} \Sigma^{+}\right)+\mathrm{H}$ ground state surface is adiabatically correlated to the $\operatorname{Cs}\left(6 \mathrm{~S}_{1 / 2}\right)+\mathrm{H}_{2}$ one, mon-adiabatic couplings necessarily occur along the reaction path. It was suggested, after calculations of potential energy ${ }^{29,30}$ and collision dynamics, ${ }^{31,32}$ that a harpooning process occurs in the entrance valley of the system, at the crossing between the neutral $\mathrm{Cs}(7 \mathrm{P})+\mathrm{H}_{2}$ entrance valley and the ionic $\mathrm{Cs}^{+}+\mathrm{H}_{2}{ }^{-}$intermediate surface. Once on this surface, the system moves toward $\mathrm{Cs}^{+} \mathrm{H}^{-}+\mathrm{H}$ products, in spite of the crossings with the ten underlying neutral potential surfaces connected with Cs atoms in the $6 \mathrm{~S}, 6 \mathrm{P}, 5 \mathrm{D}$ and 
$7 \mathrm{~S}$ states. These crossings constitute the source of efficient quenching processes which strongly limit the number of collisions leading to reaction.

A crossed-beam experiment has been devoted to the high resolution study of the reaction ${ }^{22,27,29,33}$ with a supersonic beam of hydrogen, a beam of Cs atoms and two C.W. single-mode tunable dye laser beams: The first one to excite Cs atoms over $\left(6 \mathrm{~S}_{1 / 2}, F^{\prime \prime}\right) \rightarrow\left(7 \mathrm{P}_{1 / 2,3 / 2}, F^{\prime}\right)$ hyperfine transitions and the second one to excite $\mathrm{CsH}$ products over $\left(X^{1} \Sigma^{+}\right)\left(v^{\prime \prime}=0, J^{\prime \prime}\right) \rightarrow\left(A^{1} \Sigma^{+}\right)\left(v^{\prime}=5, J^{\prime}\right)$ transitions. The fluorescence light is collected by a parabolic mirror and focussed on a low noise photomultiplier. A photon-counting chain and a microcomputer are used to record and to analyze the data. Recordings of fluorescence profiles-convolution of Doppler profiles $D\left(v-v_{0}\right)$ by the apparatus function of the set-up, a Gaussian $\approx 30 \mathrm{MHz}$ F.W.H.M.- are ensured by a step by step scanning of the laser frequency.

Since the potential energy above the reaction threshold is only $0.0016 \mathrm{eV}$ for a $\operatorname{Cs}\left(7 \mathrm{P}_{1 / 2}\right)$ excitation, the energy balance of the reaction is simply:

$$
E c \approx E j^{\prime \prime}+\Delta E
$$

where $E c$ is the kinetic energy of the reagents, $E j^{\prime \prime}$ the rotational energy of CsH products and $\Delta E$ the kinetic energy shared by the two products. The recoil velocity $w$ of $\mathrm{CsH}$ products in c.m. is:

$$
w=(1 / 135)^{1 / 2}\left(2 \Delta E / m_{\mathrm{CsH}}\right)^{1 / 2}
$$

where $m_{\mathrm{CsH}}$ is the mass of the cesium hydride. Under usual conditions, $E c$ is mainly provided by $\mathrm{H}_{2}$ molecules in their ground state level $\left(v^{\prime}=0, J^{\prime \prime}=0\right): E c=0.09 \mathrm{eV}$. Then, the reaction can populate the $\mathrm{CsH}\left(A^{1} \Sigma^{+}\right)\left(v^{\prime \prime}=0\right)$ level only, up to $J^{\prime \prime}=16$, and $w$ is small. For $J^{\prime \prime}=0, w=31 \mathrm{~m} / \mathrm{s}$ and the total Doppler width is $\Delta v_{\mathrm{D}} \approx 100$ $\mathrm{MHz}$.

The experimental results gathered on this reaction insert in the frame of the previous discussion: Selective excitation of Cs atoms and high resolution analysis of CsH product states. ${ }^{20-22,27,29,33}$

The first observation concerns the difference of reactivity between the two fine structure levels of the $7 \mathrm{P}$ state, typically $\sigma\left(7 \mathrm{P}_{1 / 2}\right) / \sigma\left(7 \mathrm{P}_{3 / 2}\right) \approx 4$ at $E c=0.09 \mathrm{eV}$. This result was interpreted in the frame of a harpooning model of collision, after hemiquantal dynamics calculations ${ }^{31}$ which showed that the parameter which determines the reactive cross section is the weight of the ${ }^{2} \Sigma$ component of the wave function, at the crossing distance $R_{\mathrm{c}}=7-8 \mathrm{a}$.u. between the neutral entrance valley and the ionic intermediate surface. It was confirmed by measuring the cross sections relative to the various hyperfine levels of the 7P state (the nuclear spin of Cs is 7/2), versus the polarization direction of the laser beam which excites Cs atoms. A rotation of the laser polarization with respect to the collision axis induces a variation of the relative population of magnetic sub-levels, thus an apparent variation of the cross section since each magnetic sub-level has a well defined reactivity (the weight of the ${ }^{2} \Sigma$ component).

As for product states, the rotational distributions in $v^{\prime \prime}=0$ were measured at two collision energies and the variations of the cross section with collision energy determined for all $J^{\prime \prime}$ values. ${ }^{22}$ 
To calculate the total reactive cross section, the number $N_{\mathrm{CsH}} / s$ of $\mathrm{CsH}$ molecules created per second in the particular quantum state $J^{\prime \prime}=11$ was first calculated from the number $N$ of photons collected during a comple scanning of the fluorescence profile. Under typical conditions, the power of the detection laser beam $\approx 50-100$ $\mathrm{mW}$ is enough to saturate the absorption and the interaction time $\Delta t=900 \mathrm{~ns}$ is long compared with the lifetime $\tau=70 \mathrm{~ns}$ of $\mathrm{CsH}$ products. The calculated Rabi frequency is $f_{1}=4.8 \mathrm{MHz}$ and the width of the interaction function $I(v)$ is $\Delta v_{\mathrm{R}}=f_{1} \sqrt{\Delta / \tau}=17 \mathrm{MHz}$, much broader than the natural width $\Gamma=2.3 \mathrm{MHz}$. The "effective" interaction function is a Lorentzian $22 \mathrm{MHz}$ F.W.H.M. and 0.7 efficiency, ${ }^{21,22} N$ and $N_{\mathrm{CsH}} / s$ are connected by the intuitive relation:

$$
N=N_{\mathrm{CsH}} / s E \xi / \delta v
$$

where $E$ is the efficiency of the detection optics $\left(E \approx 10^{-3}\right)$ and $\xi$ the area of the effective interaction function expressed in terms of the frequency steps $\delta v$. For a typical series of measurements: $N_{\mathrm{CsH}} / s=1.410^{7}, n_{\mathrm{Cs}^{*}}=7.510^{8} / \mathrm{cm}^{3}, n_{\mathrm{H} 2}=210^{12} /$ $\mathrm{cm}^{3}, v=310^{3} \mathrm{~m} / \mathrm{s}$ and $\Delta \mathrm{V}=0.8 \mathrm{~mm}^{3}$. The cross section for formation of CsH in $J^{\prime \prime}=11$ is then: $\sigma_{11}=0.3510^{-16} \mathrm{~cm}^{2}$ and the total cross section, derived by summing over all rotational product states, is: $\sigma\left(7 \mathrm{P}_{1 / 2}\right)=4.210^{-16} \mathrm{~cm}^{2}$.

This cross section is smaller than expected ${ }^{34,35}$ for a harpooning reaction, $\pi R_{\mathrm{c}}^{2} \approx 50$ $10^{-16} \mathrm{~cm}^{2}$. Since the reactive process is limited by the quenchings associated with the underlying neutral potential surfaces, the present measurement constitutes a determination of its efficiency, $\approx 10 \%$, in agreement with the prediction provided by pure quantal calculations. ${ }^{32}$

The differential cross sections were determined by use of the Doppler technique, with the two beam arrangements previously described. ${ }^{24,27}$ In spite of the small velocity of $\mathrm{CsH}$ products in c.m., profile analyses reveal a pronounced "forward" peaking of $\mathrm{CsH}$ products along the incoming $\mathrm{Cs}$ atom velocity (Figure 1). It narrows with increasing reagent velocity but is rather independent of the $J^{\prime \prime}$ values, thus of the recoil velocity of $\mathrm{CsH}$ products.

Quasi-classical trajectory calculations ${ }^{36}$ were performed from empirical three-D potential surfaces, the collinear surface being adjusted to the ab initio collinear one. ${ }^{30}$ The trajectories, initiated at the crossing between the entrance valley and the ionic intermediate, interpret the observed rotational distributions, the differential cross sections and their variations with collision energy. They show the formation of a short-lived intermediate complex and the existence of charge transfers between the two $\mathrm{H}$ atoms. In addition, they show how the initial angular momentum is conserved during the collision.

Thanks to high resolution measurements and theoretical calculations, this photochemical reaction is now well understood. The existence of a harpooning mechanism in the entrance valley is confirmed quite directly by the observation of a "hyperfine structure effect"; the measured total cross section indicates the efficiency of the reactive process. Product states are characterized for each rotational level, the shape of rotational distributions and differential cross sections being linked to the formation of a short-lived intermediate complex whose existence is shown by quasiclassical trajectory calculations. 


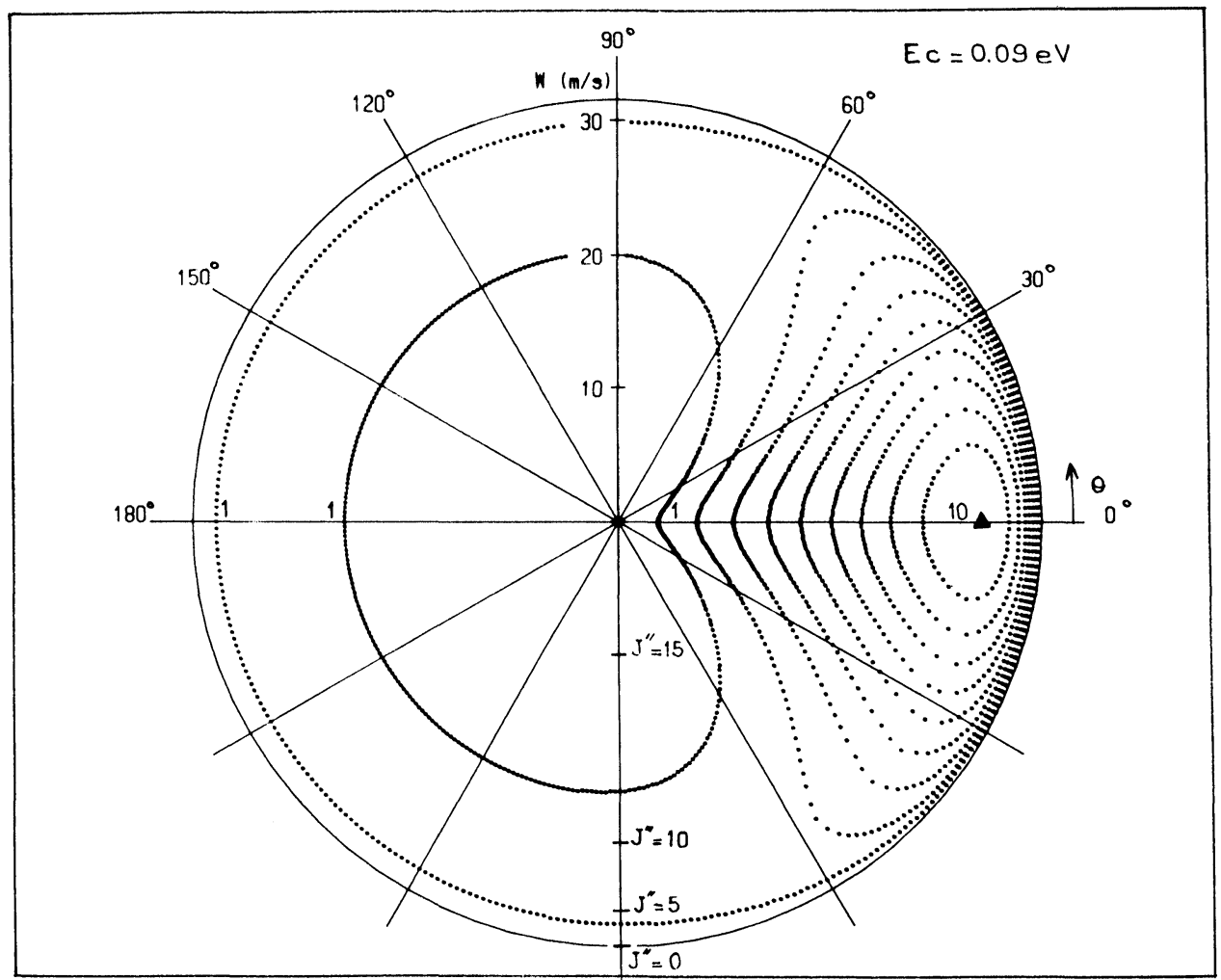

Figure 1 Centre-of-mass velocity-angle contour map, showing the probability $P(w, \Theta)$ at $E c=0.09 \mathrm{eV}$. Isoprobability contours correspond to probabilities equal to $10,9,8, \ldots$ The largest probability, 10 at $w=27 \mathrm{~m} / \mathrm{s}$ and $\Theta=0$, is marked on the figure by a black triangle.

The experiments on the $\mathrm{Cs}(7 \mathrm{P})+\mathrm{H}_{2} \rightarrow \mathrm{CsH}+\mathrm{H}$ reaction were performed with J.-M. L'hermite and G. Rahmat who are gratefully acknowledged.

\section{References}

1. Molecular Reaction Dynamics and Chemical Reactivity, ed. R. D. Levine and R. B. Bernstein (Oxford University Press, Oxford, 1987) and Chemical Dynamics via Molecular Beam and Laser Techinques, ed. R. B. Bernstein (Oxford University Press, Oxford, 1982).

2. Theoretical Aspects of Laser Radiation and its Interactions with Atomic and Molecular Systems, ed. T. F. George (N.S.F., University of Rochester, 1977) and J. Weiner, J. Chem. Phys. 72, 2856 (1980).

3. C. Jouvet, M. Boivineau, M.-C. Duval and B. Soep, J. Chem. Phys. 91, 5416 (1987) and N. F. Scherer,

L. R. Khundkar, R. B. Bernstein and A. H. Zewail, J. Chem. Phys. 87, 145 (1987).

4. P. Hering, P. R. Brooks, R. F. Curl, R. S. Judson and R. S. Lowe, Phys. Rev. Lett. 44, 687 (1980) and P. Arrowsmith, S. H. Bly, P. E. Charters and J. C. Polanyi, J. Chem. Phys. 79, 283 (1983).

5. A. H. Zewail, Science 242, 1645 (1988) and J. Chem. Soc., Faraday Trans. II 85(8), 1221 (1989).

6. P. J. Dagdigian and M. L. Campbell, Chemical Reviews 87, 1 (1987) and D. Husain and G. Roberts, in Bimolecular Collisions, ed. M. N. R. Ashfold and J. E. Baggott (R.S.C., London, 1989).

7. S. Hayashi, T. M. Mayer and R. B. Bernstein, Chem. Phys. Lett. 53, 419 (1978).

8. H. K. Haugen, E. Weitz and S. R. Leone, Chem. Phys. Lett. 119, 75 (1985). 
9. P. S. Weiss, J.-M. Mestdagh, H. Schmidt, M. f. Vernnon, M. H. Covinsky, B. A. Balko and Y. T. Lee, in Recent Advances in Molecular Reaction Dynamics, ed. R. Vetter and J. Vigué (C.N.R.S., Paris, 1986).

10. J.-M. Mestdagh, C. Alcaraz, J. Berlande, J. Cuvellier, T. Gustavsson, P. Meynadier, P. de Pujo, O. Sublemontier and J.-P. Visticot, Laser Chem. 10, 389 (1990) and C. Alcaraz, Thèse de Doctorat, Université d'Orsay (1990).

11. E. Verdasco and A. Gonzalez-Urena, J. Chem. Phys. 93, 428 (1990).

12. J. C. Polanyi and W. H. Wong, J. Chem. Phys. 51, 1439 (1969).

13. D. Arnoldi and J. Wolfrum, Phys. Chem. 80, 892 (1976).

14. T. J. Odiorne, P. R. Brooks and J. V. Kasper, J. Chem. Phys. 55, 1980 (1971).

15. H. J. Loesch in Laseres y Reacciones Quimicas, ed. A. Gonzalez-Urena (Cursos de Verano, Universidad Complutense de Madrid, Madrid, 1989).

16. U. Gaubatz, P. Rudecki, M. Becker, S. Schiemann, M. Kulz and K. Bergmann, Chem. Phys. Lett. 149, 463 (1989).

17. C. T. Rettner and R. N. Zare, J. Chem. Phys. 77, 2416 (1982).

18. Orientation and Polarisation Effects in Reactive Collisions, J. Chem. Soc., Faraday Trans. II 85(8) (1989).

19. N. Billy, B. Girard, G. Gouédard, J. Vigué, Mol. Phys. 61, 65 (1987); Laser Chem. 10, 319 (1990) and

B. Girard, Thèse de Doctorat, Université de Paris (1987).

20. J.-M. L'Hermite, G. Rahmat and R. Vetter, Laser Chem. 10, 377 (1990).

21. J.-M. L'Hermite, Thèse de Doctorat, Université d'Orsay (1990).

22. J.-M. L'Hermite, G. Rahmat and R. Vetter, J. Chem. Phys. in press (1991).

23. J. L. Kinsey, J. Chem. Phys. 66, 2560 (1977).

24. J. A. Serri, J. L. Kinsey and D. E. Pritchard, J. Chem. Phys. 75, 663 (1981).

25. W. D. Phillips, J. A. Serri, D. J. Ely, D. E. Pritchard, K. R. Way and J. L. Kinsey, Phys. Rev. Lett. 41, 937 (1978); J. A. Serri, C. H. Becker, M. B. Elbel, J. L. Kinsey, W. P. Moscowitz and D. E. Pritchard, J. Chem. Phys. 74, 5116 (1981) and W. P. Moscowitz, B. Stewart, R. M. Bilotta, J. L. Kinsey, D. E. Pritchard, J. Chem. Phys. 80, 5496 (1984).

26. E. J. Murphy, J. H. Brophy, G. S. Arnold, W. L. Dimpfl and J. L. Kinsey, J. Chem. Phys. 70, 5910 (1979).

27. J.-M. L'Hermite, G. Rahmat and R. Vetter, J. Chem. Phys. 93, 434 (1990).

28. B. Girard, N. Billy, G. Gouédard and J. Vigué, Europhysics Lett. 14, 13 (1991).

29. C. Crépin, J.-L. Picqué, G. Rahmat, J. Vergès, R. Vetter, F.-X Gadéa, M. Pélissier, F. Spiegelmann and J.-P. Malrieu, Chem. Phys. Lett. 110, 395 (1984) and G. Rahmat, J. Vergès, R. Vetter, F.-X Gadéa, M. Pélissier, F. Spiegelmann, in Recent Advances in Molecular Reaction Dynamics, ed. R. Vetter and J. Vigué (C.N.R.S., Paris, 1986).

30. F.-X Gadéa, F. Spiegelmann, M. Pélissier and J.-P. Malrieu, J. Chem. Phys. 84, 4872 (1986).

31. F.-X Gadéa and J. Durup, Chem. Phys. Lett. 138, 43 (1987).

32. B. Lepetit, M. Le Dourneuf, J.-M. Launay and F.-X Gadéa, Chem. Phys. Lett. 135, 377 (1987). B. Lepetit, J.-M. Launay and M. Le Dourneuf, Chem. Phys. 134, 1 (1989).

33. G. Rahmat, F. Spiegelmann, J. Vergès and R. Vetter, Chem. Phys. Lett. 135, 459 (1987); G. Rahmat, J. Vergès and R. Vetter, J. de Phys. Supp. 12 48, 601 (1987) and F.-X Gadéa, J.-M. L'Hermite, G. Rahmat and R. Vetter, Chem. Phys. Lett. 151, 183 (1988).

34. D. R. Herschbach, Appl. Opt. Supp. 2, 128 (1965).

35. Alkali Halide Vapors, ed. P. Davidovits and D. L. MacFadden (Academic Press, New York, 1979).

36. J.-M. L'Hermite, to be published (1991). 